\title{
Does Stroke Severity for Repeated Thrombolysis Matter? Response to the Letter by Wu et al.
}

\author{
Mona Laible $^{\mathrm{a}}$ Ekkehart Jenetzky ${ }^{\mathrm{b}-\mathrm{d}}$ Peter Arthur Ringleb ${ }^{\mathrm{a}} \quad$ Timolaos Rizos $^{\mathrm{a}}$ \\ a Department of Neurology, University of Heidelberg, and ${ }^{b}$ Division of Clinical Epidemiology and Aging Research, \\ German Cancer Research Center, Heidelberg, ' Department for Child and Adolescent Psychiatry, Johannes \\ Gutenberg-University, Mainz, and ${ }^{\mathrm{d}}$ Child Center Maulbronn gGmbH, Hospital for Paediatric Neurology and Social \\ Paediatrics, Maulbronn, Germany
}

Dear Sir,

We appreciate Dr. Wu's interest in our article $[1,2]$. By analyzing a comparatively large cohort of ischemic stroke patients repeatedly treated with tissue-type plasminogen activator (rt-PA) at a single center and by applying standard treatment procedures and outcome measures, we observed that repeated thrombolysis was not associated with a higher rate of complications compared to complications that arose as a result of the first treatment, but our data indicated that the clinical outcome after the second treatment appeared not to be as satisfactory as the outcome of the first treatment [1]. Although our study was retrospective in design, onset-to-treatment times for the second thrombolysis were known in $87.5 \%$ and interinfarct times were available in all patients [1]. However, due to the limited size of our cohort, multivariate analysis was not reasonable.

Drs. Wu, Huang and Ma raise the issue that no larger-scale multicenter studies evaluating safety, efficacy and outcome effects in ischemic stroke patients repeatedly treated with rt-PA are existent. We have several concerns regarding their analyses.

Although they criticized on the number of patients with missing onset to treatment-times in our cohort, they included these cases and conclude from their analy- sis that the initial stroke severity would predict functional outcome and ICH after repeated thrombolytic treatment. It is important to note here that comparability of the findings of previous studies on repeated thrombolytic treatment is highly limited. At first, definitions of clinical outcome considerably varied among these reports. As a consequence, $\mathrm{Wu}$ et al. defined a good clinical outcome in an inhomogeneous way: in some cases, good clinical outcome was defined as a change in NIHSS (described as a progress (?) in NIHSS $\geq 4$ ) and in other cases by an mRS $\leq 2$ at 3 months. Second, definitions used to report ICH after thrombolytic treatment also varied substantially among previous reports [3-6]. Nevertheless, supposing comparability, $\mathrm{Wu}$ et al. entered these reported ICH results into their model. We do have additional concerns with regard to the subgroup calculation performed in patients repeatedly thrombolysed within 30 days after a first treatment: according to $\mathrm{Wu}$ et al., the rate of $\mathrm{ICH}$ in this subgroup was $25 \%$. Apparently, repeated bleeding events in identical subjects who received a third and fourth thrombolytic treatment were added as a single bleeding event. From a statistical point of view, this approach is incorrect. Against this background and because the 15 observational studies that were used in- cluded on average less than 4 cases, the 2 logistic regression analyses appear not appropriate from our point of view. Using the presented dataset, we moreover could not reproduce the ORs concerning the impact of NIHSS values on a good functional outcome and the impact of NIHSS values on $\mathrm{ICH}$.

To conclude, in our opinion, available data on repeated treatment with rt-PA do not permit defining any clinical guidance. Future research should focus on larger populations and analyze predefined standardized variables to enable multivariate comparisons to evaluate for risk factors associated with poor outcome after repeated treatment with rt-PA.

\section{Disclosure Statement}

M.L. is supported by a fellowship of the Medical Faculty, University of Heidelberg. T.R. has received consulting honoraria, speakers' honoraria and travel support from BMS Pfizer, Boehringer Ingelheim, Bayer HealthCare and Daiichi Sankyo. E.J. reports no disclosures. P.A.R. has received consulting honoraria, speakers' honoraria and travel support from BMS Pfizer, Boehringer Ingelheim, Bayer HealthCare and Daiichi Sankyo.

\section{KARGER}

E-Mail karger@karger.com

www.karger.com/ene
(C) 2016 S. Karger AG, Base

0014-3022/16/0754-0155\$39.50/0
Timolaos Rizos, MD

Department of Neurology, University of Heidelberg

Im Neuenheimer Feld 400

DE-69120 Heidelberg (Germany)

E-Mail Timolaos.Rizos@med.uni-heidelberg.de 


\section{References}

1 Laible M, Jenetzky E, Möhlenbruch M, Ringleb PA, Rizos T: Repeated intravenous treatment with recombinant tissue-type plasminogen activator in patients with acute ischemic stroke. Eur Neurol 2015;74:127-134.

2 Wu J, Huang Q, Ma Q: What matters in the results of repeated intraveno us thrombolysis for recurrent ischemic stroke? Eur Neurol 2016;75:150-154.
3 Yoo HS, Kim YD, Lee HS, et al: Repeated thrombolytic therapy in patients with recurrent acute ischemic stroke. J Stroke 2013;15: $182-188$.

4 Sposato LA, Salutto V, Beratti DE, et al: Adverse outcome of early recurrent ischemic stroke secondary to atrial fibrillation after repeated systemic thrombolysis. Case Rep Vasc Med 2013;2013:371642.
5 Cappellari M, Moretto G, Bovi P: Repeated intravenous thrombolysis after recurrent stroke. A case series and review of the literature. J Neurol Sci 2014;345:181-183.

6 Sauer R, Huttner HB, Breuer L, et al: Repeated thrombolysis for chronologically separated ischemic strokes: a case series. Stroke 2010;41: 1829-1832. 